\title{
Multi-channel tri-gate normally-on/off AIGaN/GaN MOSHEMTs on Si substrate with high breakdown voltage and low ON-resistance
}

\author{
Jun $\mathrm{Ma},{ }^{1, a)}$ Catherine Erine, ${ }^{1}$ Peng Xiang, ${ }^{2}$ Kai Cheng, ${ }^{2}$ and Elison Matioli ${ }^{1, b)}$ \\ ${ }^{1}$ Power and Wide-Band-Gap Electronics Research Laboratory, École Polytechnique Fédérale de Lausanne \\ (EPFL), CH-1015 Lausanne, Switzerland \\ ${ }^{2}$ Enkris Semiconductor Inc., Suzhou 215123, People's Republic of China
}

(Received 4 October 2018; accepted 19 November 2018; published online 10 December 2018)

\begin{abstract}
In this work, we present multi-channel tri-gate $\mathrm{AlGaN} / \mathrm{GaN}$ metal-oxide-semiconductor high-electron-mobility transistors (MOSHEMTs) for high-voltage applications. A heterostructure with multiple $\mathrm{AlGaN} / \mathrm{GaN}$ layers was used to form five parallel two-dimensional-electron-gas (2DEG) channels to reduce the $\mathrm{ON}$-resistance $\left(R_{\mathrm{ON}}\right)$, simultaneously modulated by the 3-dimensional trigate electrodes. The tri-gate is a unique technology to control the multi-channels, providing enhanced electrostatics and device performance, and, in turn, the multi-channels are exceptionally suited to address the degradation in drain current $\left(I_{\mathrm{D}, \max }\right)$ caused by the tri-gate. With a tri-gate width $(w)$ of $100 \mathrm{~nm}$, normally-on multi-channel tri-gate transistors presented $3 \times$-higher maximum drain current $\left(I_{\mathrm{D}, \max }\right), 47 \%$-smaller $R_{\mathrm{ON}}$, as well as $79 \%$-higher maximum transconductance $\left(g_{\mathrm{m}, \max }\right)$, as compared to counterpart single-channel devices. Using the channel depletion through the tri-gate sidewalls, normally-off operation was also achieved by reducing $w$ below the sidewall depletion width $\left(w_{\text {dep }}\right)$, resulting in a positive threshold voltage $\left(V_{\mathrm{TH}}\right)$ of $0.82 \mathrm{~V}$ at $1 \mu \mathrm{A} / \mathrm{mm}$. The devices presented a high breakdown voltage $\left(V_{\mathrm{BR}}\right)$ of $715 \mathrm{~V}$, which reveals a promising future platform for high-voltage low- $R_{\mathrm{ON}} \mathrm{GaN}$ transistors. Published by AIP Publishing.
\end{abstract}

https://doi.org/10.1063/1.5064407

The exceptional properties of $\mathrm{GaN}$ heterostructures have led to the development of $\mathrm{AlGaN} / \mathrm{GaN}$ high electron mobility transistors (HEMTs), demonstrating outstanding potential for high-voltage applications. ${ }^{1-5}$ Nevertheless, the performance of current GaN HEMTs is still far below the prospect promised by this material. Further improvements require a significant reduction in $R_{\mathrm{ON}}$, increase in $V_{\mathrm{BR}}$, while maintaining good performance at high switching frequencies.

Among these factors, $R_{\mathrm{ON}}$ is intrinsically determined by the electric conductivity of the two-dimensional-electron-gas (2DEG) channel at the $\mathrm{AlGaN} / \mathrm{GaN}$ interface, given by the product of its sheet carrier concentration $\left(N_{\mathrm{s}}\right)$ and mobility $(\mu)$. High-Al-content barrier materials have been intensively explored to increase the $N_{\mathrm{s}}$, which however results in a reduced $\mu$ in addition to a more challenging epitaxial growth. ${ }^{6-9}$ Moreover, the increased $N_{\mathrm{s}}$ yields a more negative $V_{\mathrm{TH}}$ and makes it much more difficult to achieve normally-off operation.

To address these challenges, we present here multi-channel tri-gate metal-oxide-semiconductor high-electron-mobility transistors (MOSHEMTs) [Fig. 1(a)]. This unique architecture offers smaller $R_{\mathrm{ON}}$, due to the large $N_{\mathrm{s}}$ and high $\mu$ in the multichannels, ${ }^{10-12}$ and superior gate control with the 3-dimensional tri-gate structure [Fig. 1(b)], integrating multiple parallel transistors in a given footprint for enhanced performance and reduced substrate cost [Fig. 1(c)]. Here, we demonstrate these devices [Fig. 1(d)] using a five-channel AlGaN/GaN-on-Si heterostructure, investigate the impact of tri-gate geometry on the device performance, and present multi-channel tri-gate devices with high breakdown voltage and low $R_{\mathrm{ON}}$.

\footnotetext{
${ }^{a)}$ E-mail: jun.ma@epfl.ch

${ }^{\text {b) }}$ E-mail: elison.matioli@epfl.ch
}

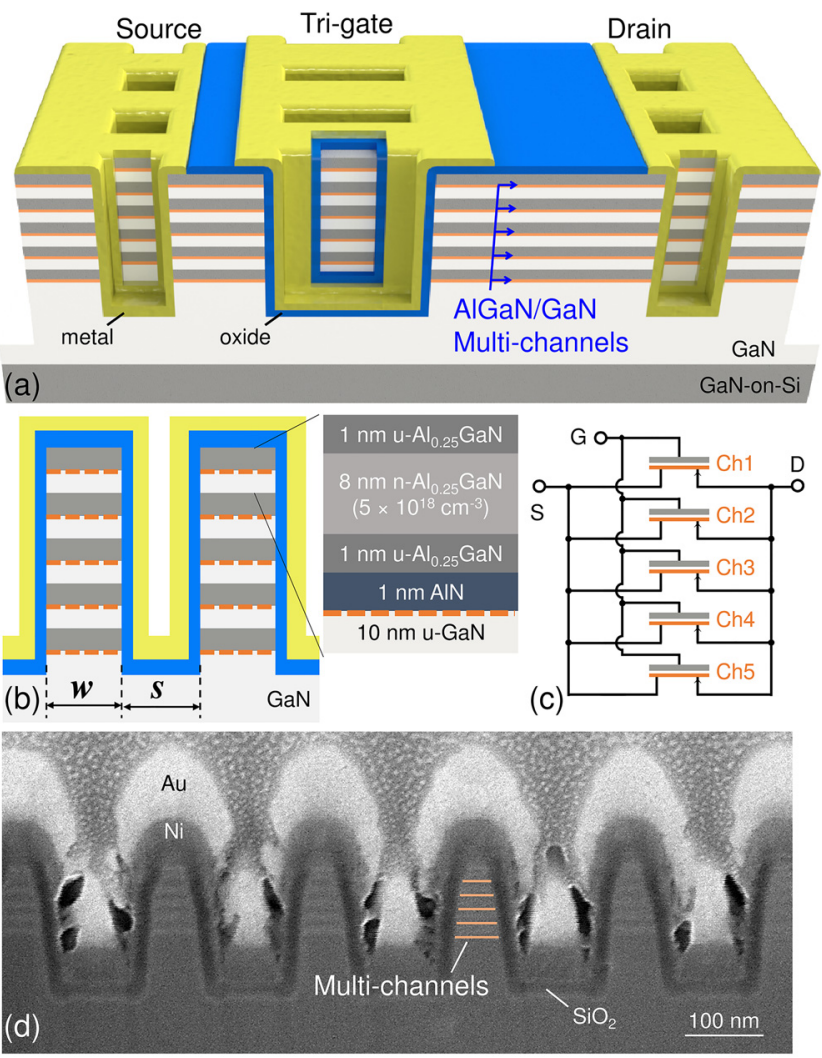

FIG. 1. (a) Schematic of the multi-channel tri-gate $\mathrm{AlGaN} / \mathrm{GaN}$ MOSHEMT. (b) Cross-sectional schematic of the tri-gate region. The inset shows the heterostructure forming each of the multi-channels. (c) An equivalent circuit of the multi-channel tri-gate MOSHEMT, integrating multiple parallel transistors in a given device footprint for enhanced performance and reduced substrate cost. (d) A cross-sectional SEM image of the tri-gate region, tilted by $52^{\circ}$. 
The multi-channel AlGaN/GaN-on-Si heterostructure in this work consisted of 5 parallel 2DEG channels, formed by a $10 \mathrm{~nm}$-thick AlGaN barrier, a $1 \mathrm{~nm}$-thick AlN spacer, and a $10 \mathrm{~nm}$-thick GaN channel layers. The barrier layer was partially doped with $\mathrm{Si}$ at $5 \times 10^{18} \mathrm{~cm}^{-3}$ [Fig. 1(b)] to enhance the conductivity of multi-channel $\mathrm{AlGaN} / \mathrm{GaN}$ heterostructures, especially with thin $\mathrm{AlGaN}$ barrier layers. Hall measurements revealed a small sheet resistance $\left(R_{\mathrm{S}}\right)$ of $230 \Omega / \mathrm{sq}$, $N_{\mathrm{s}}$ of $1.5 \times 10^{13} \mathrm{~cm}^{-2}$, and $\mu$ of $1820 \mathrm{~cm}^{2} \mathrm{~V}^{-1} \mathrm{~s}^{-1}$. A small effective resistivity ( $\left.\rho_{\text {eff }}\right)$ of $2.4 \mathrm{~m} \Omega \mathrm{cm}$ was obtained, comparable to other literature results, ${ }^{13-19}$ but with a small total thickness ( $\left.t_{\text {tot }}\right)$ and higher $\mu$ (Fig. 2). Small $\rho_{\text {eff }}$ and high $\mu$ are crucial to reduce $R_{\mathrm{ON}}$, and a thin $t_{\text {tot }}$ facilitates electrostatic gate control and device fabrication (the etching of high-aspect ratio fins and the formation of electrodes around them can be challenging).

The device fabrication started with e-beam lithography to define the fins, which were etched by $\mathrm{Ar} / \mathrm{Cl}_{2}$-based inductively coupled plasma (ICP) with a depth of $200 \mathrm{~nm}$. The fins in the tri-gate region had different $w$, spacings $(s)$, filling factors $(F F)$, and lengths $(l)$, while the fins in ohmic regions had $w$ and $s$ of $500 \mathrm{~nm}$ to contact the multi-channels. Then, an ohmic stack of $\mathrm{Ti} / \mathrm{Al} / \mathrm{Ti} / \mathrm{Ni} / \mathrm{Au}$ was formed in source and drain regions by ebeam evaporation and a lift-off process, followed by rapid thermal annealing at $800^{\circ} \mathrm{C}$ in nitrogen. $25 \mathrm{~nm}$ of $\mathrm{SiO}_{2}$ was deposited by atomic layer deposition (ALD) at $300^{\circ} \mathrm{C}$ as the gate dielectric, which was selectively removed by diluted HF (1\%) in ohmic regions to expose the source and drain contacts. Finally, the gate was formed using Ni/Au. All current values in this work were normalized by the width of device footprint, unless specified, and the standard deviation was determined from measurements of about 12 devices of each type.

Tri-gates are uniquely suited to control the multichannels. To illustrate this, we fabricated multi-channel trigate transistors with different $w$ but with a long fixed $l$ of $50 \mu \mathrm{m}$, which diminished the impact from access and ohmic regions on the extracted device characteristics. Here, the $I_{\mathrm{D}}$ and $g_{\mathrm{m}}$ were normalized by the total width of the long fins, which in this case dominated the device characteristics. More details about these devices can be found in the caption of Fig. 3 as well as Table I. The MOS channels at trenches

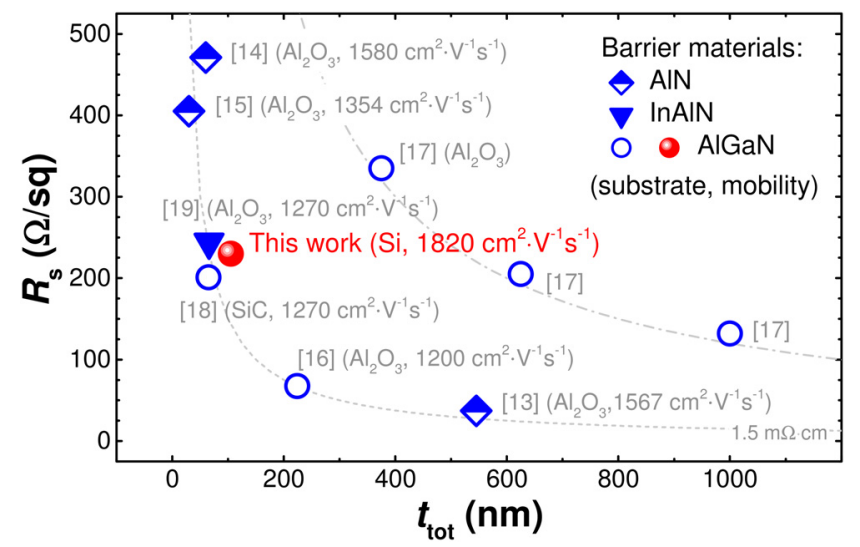

FIG. 2. Comparison of multi-channel structures in this work with literature results. The $t_{\text {tot }}$ refers to the total thickness of the multi-channel structure. For a fair comparison, multi-channel structures with unspecified $R_{\mathrm{s}}$ were not included. $^{20,21}$
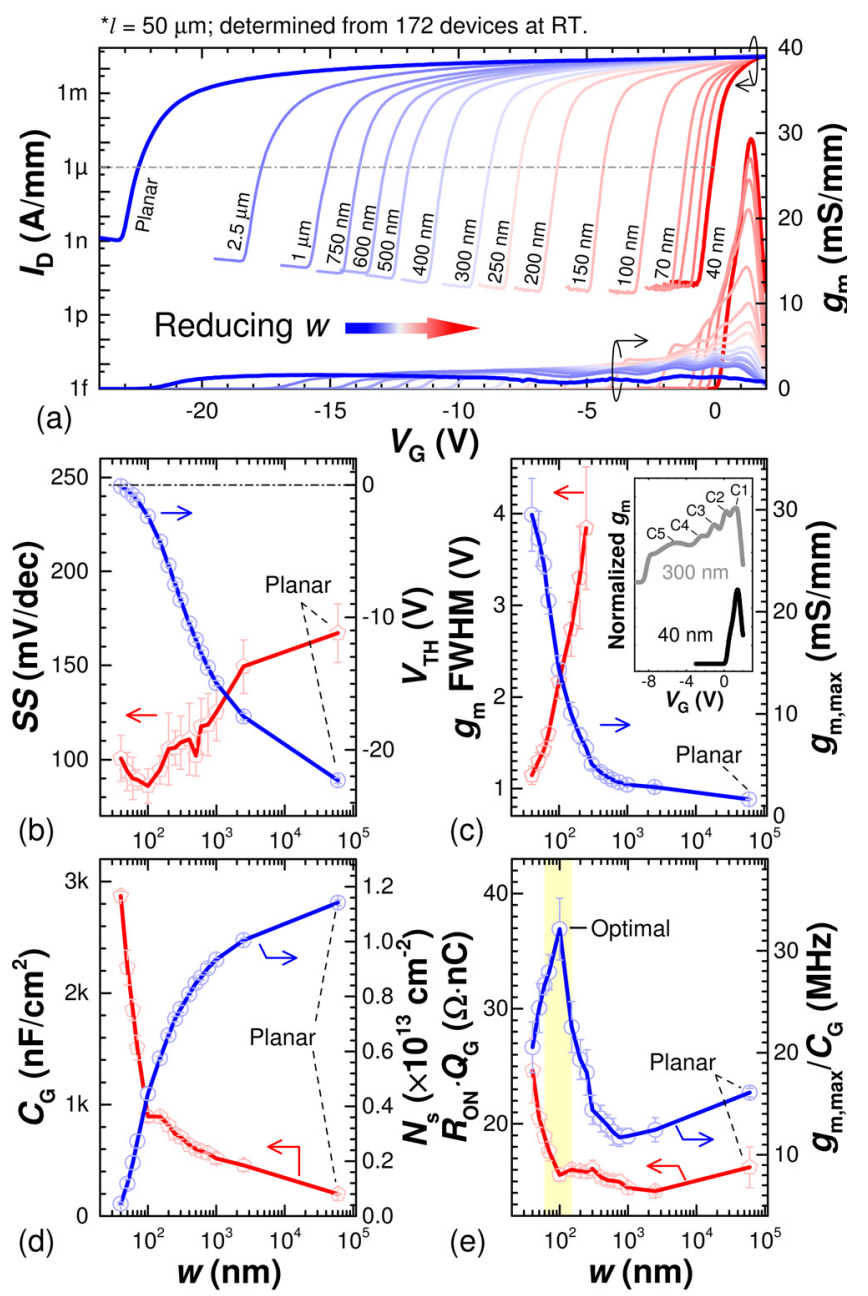

FIG. 3. (a) Transfer characteristics of multi-channel tri-gate MOSHEMTs with different $w$ from $60 \mu \mathrm{m}$ (planar) to $40 \mathrm{~nm}$, measured at $V_{\mathrm{D}}=0.5 \mathrm{~V}$. Width dependence of (b) $S S$ and $V_{\mathrm{th}}$ (at $\left.1 \mu \mathrm{A} / \mathrm{mm}\right)$, (c) $g_{\mathrm{m}, \max }$ and $g_{\mathrm{m}}$ FWHM, (d) $C_{\mathrm{G}}$ and $N_{\mathrm{s}}$, and (e) $R_{\mathrm{ON}} \cdot Q_{\mathrm{G}}\left(Q_{\mathrm{G}}\right.$ refers to gate charge) and $g_{\mathrm{m}, \max } / C_{\mathrm{G}}$ values. The inset in (c) shows normalized $g_{\mathrm{m}}-V_{\mathrm{G}}$ plots of devices with $w$ of $40 \mathrm{~nm}$ and $300 \mathrm{~nm}$. The $R_{\mathrm{ON}}, C_{\mathrm{G}}$, and $Q_{\mathrm{G}}$ were extracted at $V_{\mathrm{G}}=2 \mathrm{~V}, N_{\mathrm{s}}$ was extracted at $V_{\mathrm{G}}=0 \mathrm{~V}$, and the $g_{\mathrm{m}, \max }$ was the maximum value of the $g_{\mathrm{m}}-V_{\mathrm{G}}$ characteristics, regardless of the several $g_{\mathrm{m}}$ peaks in transistors with $w>200 \mathrm{~nm}$. The $C_{\mathrm{G}}$ and $N_{\mathrm{s}}$ were normalized by the top surface area of the fins. The length of the gate electrode here $\left(L_{\mathrm{G}}\right)$ was $51 \mu \mathrm{m}$, covering the fins and extending $0.5 \mu \mathrm{m}$ towards the source and drain. The gate-to-source $\left(L_{\mathrm{GS}}\right)$ and gate-to-drain $\left(L_{\mathrm{GD}}\right)$ lengths were $1.5 \mu \mathrm{m}$.

were not considered since the measurements were conducted with $V_{\mathrm{G}}$ below the $V_{\mathrm{TH}}$ of the MOS channel, which was $\sim 2 \mathrm{~V}$ for our oxides and fabrication process, ${ }^{22}$ and the conductivity of the MOS channel is much smaller than that of the 2DEG channels. ${ }^{22}$

Figure 3(a) shows the impact of reducing $w$ on the transfer characteristics of the devices. Conventional planar gates are not suited to electrostatically control the multi-channel structure, which is indicated by their large $V_{\mathrm{TH}}$ of $-22.3 \pm 0.2 \mathrm{~V}$ (at 1 $\mu \mathrm{A} / \mathrm{mm}$ ), poor subthreshold swing (SS) of $167 \pm 16 \mathrm{mV} / \mathrm{dec}$, and small $g_{\mathrm{m}, \max }$ of $1.63 \pm 0.04 \mathrm{mS} / \mathrm{mm}$. The large $\left|V_{\mathrm{TH}}\right|$ is caused by the large gate-to-channel distance and the screening effect that shields a lower channel from the gate control unless its upper channel is depleted. The small $g_{\mathrm{m}, \max }$ and the large $S S$ indicate that control of the multi-channels is not simultaneous. The tri-gate addresses this issue by providing additional electrostatic control from its sidewall portions, which can be 
TABLE I. Tri-gate geometry and device characteristics for the $50 \mu \mathrm{m}$-long multi-channel tri-gate transistors presented in Fig. 3 . The $N_{\mathrm{s}}$ here was normalized by the top surface area of the fins.

\begin{tabular}{|c|c|c|c|c|c|c|c|c|c|c|c|c|}
\hline $\begin{array}{l}w \\
(\mathrm{~nm})\end{array}$ & $\begin{array}{c}s \\
(\mathrm{~nm})\end{array}$ & $\begin{array}{l}F F \\
(\%)\end{array}$ & $\begin{array}{c}l \\
(\mu \mathrm{m})\end{array}$ & $\begin{array}{l}\text { Number } \\
\text { of fins }\end{array}$ & $\begin{array}{l}\text { Total width } \\
\text { of fins }(\mu \mathrm{m})\end{array}$ & $\begin{array}{c}V_{\mathrm{TH}} \\
@ I_{\mathrm{D}}=1 \mu \mathrm{A} / \mathrm{mm} \\
(\mathrm{V})\end{array}$ & $\begin{array}{c}S S \\
@ V_{\mathrm{D}}=0.5 \mathrm{~V} \\
(\mathrm{mV} / \mathrm{dec})\end{array}$ & $\begin{array}{c}g_{\mathrm{m}, \max } \\
@ V_{\mathrm{D}}=0.5 \mathrm{~V} \\
(\mu \mathrm{S})\end{array}$ & $\begin{array}{c}R_{\mathrm{ON}} \\
@ V_{\mathrm{G}}=2 \mathrm{~V} \\
(\Omega)\end{array}$ & $\begin{array}{c}C_{\mathrm{G}} \\
@ V_{\mathrm{G}}=2 \mathrm{~V} \\
(\mathrm{pF})\end{array}$ & $\begin{array}{c}\mathrm{Q} \\
V_{\mathrm{G}}=2 \mathrm{~V} \\
(\mathrm{pC})\end{array}$ & $\begin{array}{c}N_{\mathrm{s}} \\
@ V_{\mathrm{G}}=0 \mathrm{~V} \\
\left(10^{13} \mathrm{~cm}^{-2}\right)\end{array}$ \\
\hline 40 & 160 & 20 & 50 & 300 & 12 & $-0.08 \pm 0.04$ & $101 \pm 12$ & $354 \pm 43$ & $1212 \pm 134$ & $17.2 \pm 0.23$ & $20.3 \pm 0.6$ & $0.05 \pm 0.01$ \\
\hline 50 & 150 & 25 & 50 & 300 & 15 & $-0.42 \pm 0.04$ & $93.7 \pm 10$ & $408 \pm 40$ & $929 \pm 60$ & $16.7 \pm 1.2$ & $22.2 \pm 1.8$ & $0.12 \pm 0.02$ \\
\hline 60 & 140 & 30 & 50 & 300 & 18 & $-0.78 \pm 0.04$ & $90.0 \pm 11$ & $443 \pm 16$ & $769 \pm 47$ & $16.6 \pm 0.84$ & $24.6 \pm 1.7$ & $0.19 \pm 0.02$ \\
\hline 70 & 130 & 35 & 50 & 300 & 21 & $-1.14 \pm 0.05$ & $89.4 \pm 5.3$ & $442 \pm 27$ & $664 \pm 19$ & $15.9 \pm 1.1$ & $26.5 \pm 1.5$ & $0.27 \pm 0.03$ \\
\hline 100 & 100 & 50 & 50 & 300 & 30 & $-2.37 \pm 0.09$ & $86.0 \pm 9.2$ & $430 \pm 41$ & $487 \pm 13$ & $13.4 \pm 0.69$ & $32.0 \pm 1.3$ & $0.45 \pm 0.02$ \\
\hline 150 & 150 & 50 & 50 & 200 & 30 & $-4.28 \pm 0.14$ & $94.5 \pm 7.6$ & $301 \pm 33$ & $487 \pm 8.4$ & $13.4 \pm 0.32$ & $32.9 \pm 0.7$ & $0.58 \pm 0.01$ \\
\hline 200 & 200 & 50 & 50 & 150 & 30 & $-6.08 \pm 0.12$ & $106 \pm 19$ & $264 \pm 24$ & $486 \pm 11$ & $12.2 \pm 0.18$ & $32.7 \pm 0.4$ & $0.66 \pm 0.01$ \\
\hline 250 & 250 & 50 & 50 & 120 & 30 & $-7.52 \pm 0.15$ & $106 \pm 9.1$ & $200 \pm 25$ & $489 \pm 8.2$ & $11.1 \pm 0.59$ & $32.4 \pm 0.6$ & $0.72 \pm 0.01$ \\
\hline 300 & 300 & 50 & 50 & 100 & 30 & $-8.69 \pm 0.14$ & $109 \pm 14$ & $153 \pm 15$ & $499 \pm 21$ & $10.6 \pm 0.28$ & $32.2 \pm 0.9$ & $0.75 \pm 0.01$ \\
\hline 400 & 400 & 50 & 50 & 75 & 30 & $-10.5 \pm 0.13$ & $111 \pm 19$ & $130 \pm 12$ & $483 \pm 8.1$ & $9.59 \pm 0.34$ & $31.8 \pm 0.7$ & $0.81 \pm 0.01$ \\
\hline 500 & 500 & 50 & 50 & 60 & 30 & $-11.7 \pm 0.18$ & $102 \pm 11$ & $116 \pm 9.6$ & $473 \pm 13$ & $9.03 \pm 0.48$ & $32.0 \pm 0.6$ & $0.85 \pm 0.01$ \\
\hline 600 & 600 & 50 & 50 & 50 & 30 & $-12.7 \pm 0.17$ & $118 \pm 15$ & $107 \pm 11$ & $470 \pm 7.3$ & $8.76 \pm 0.33$ & $32.0 \pm 0.9$ & $0.87 \pm 0.01$ \\
\hline 750 & 750 & 50 & 50 & 40 & 30 & $-13.8 \pm 0.13$ & $119 \pm 17$ & $99.3 \pm 7.5$ & $463 \pm 15$ & $8.48 \pm 0.15$ & $32.3 \pm 0.6$ & $0.90 \pm 0.01$ \\
\hline 1000 & 1000 & 50 & 50 & 30 & 30 & $-14.9 \pm 0.20$ & $125 \pm 15$ & $92.2 \pm 5.8$ & $449 \pm 8.6$ & $7.78 \pm 0.66$ & $32.2 \pm 0.6$ & $0.93 \pm 0.01$ \\
\hline 2500 & 2500 & 50 & 50 & 12 & 30 & $-17.5 \pm 0.24$ & $149 \pm 14$ & $85.1 \pm 8.1$ & $430 \pm 11$ & $6.84 \pm 0.80$ & $32.9 \pm 0.6$ & $1.01 \pm 0.01$ \\
\hline Planar & 0 & 100 & 51 & 1 & 60 & $-22.3 \pm 0.20$ & $167 \pm 16$ & $81.3 \pm 2.0$ & $239 \pm 26$ & $6.06 \pm 0.12$ & $67.8 \pm 0.6$ & $1.14 \pm 0.01$ \\
\hline
\end{tabular}

enhanced by reducing $w,{ }^{23-25}$ leading to much improved $V_{\mathrm{TH}}$ and $S S$ [Fig. 3(b)]. At $w$ of $40 \mathrm{~nm}$, the channel control is dominated by the sidewalls over the top gate, resulting in small $V_{\mathrm{TH}}$ of $-0.08 \pm 0.04 \mathrm{~V}$ and improved $S S$ of $101 \pm 12 \mathrm{mV} / \mathrm{dec}$. The multi-channels are also modulated simultaneously by the trigate, as revealed by the $g_{\mathrm{m}}-V_{\mathrm{G}}$ characteristics [Fig. 3(c)]. In transistors with planar gates or wide tri-gates $(w>200 \mathrm{~nm})$, the $g_{\mathrm{m}}$ shows clearly five separate peaks, caused by the successive turn on of each of the five channels [inset in Fig. 3(c)]. By reducing $w$, these peaks merge and their full width at half maximum (FWHM) is reduced, forming finally a single sharp peak at $w$ of $40 \mathrm{~nm}$ with high $g_{\mathrm{m}, \max }$ of $29.5 \pm 3.6 \mathrm{mS} / \mathrm{mm}$. This is because the $V_{\mathrm{TH}}$ of each parallel channel is mainly determined by the sidewall control. Thus all channels turn on simultaneously. The enhanced electrostatic control is due to the increased gate capacitance $\left(C_{\mathrm{G}}\right)$ and reduced $N_{\mathrm{s}}$ with narrowing tri-gates [Fig. 3(d)]. Such an increase in $C_{\mathrm{G}}$ does not necessarily degrade the transistor frequency performance. An optimized $w$ of $100 \mathrm{~nm}$ for multi-channel tri-gate devices led to similar $R_{\mathrm{ON}} \cdot Q_{\mathrm{G}}$ product $(15.6 \pm 0.05 \Omega \mathrm{nC})$ and twice the $g_{\mathrm{m}, \max } / C_{\mathrm{G}}$ value $(32.1 \pm 0.3 \mathrm{MHz})$ as compared with planar-gate devices $(16.2 \pm 1.77 \Omega \mathrm{nC}$ and $16.1 \pm 0.04 \mathrm{MHz})$, suggesting an enhanced frequency performance [Fig. 3(e)].

While tri-gates are uniquely adapted to control the multi-channels, the multi-channel structure is exceptionally suited to address the degraded $I_{\mathrm{D} \text {, max }}$ caused by the tri-gate. As shown in Fig. 4(a), $I_{\mathrm{D}, \max }$ was greatly reduced by $17 \%$ in single-channel devices even at a large $w$ of $1 \mu \mathrm{m}$, and such reduction further increased to $41 \%$ as $w$ was reduced to $100 \mathrm{~nm}$. In contrast, the $I_{\mathrm{D}, \max }$ in multi-channel devices reduced only by $6 \%$ at $w$ of $1 \mu \mathrm{m}$, and remained constant until $w$ of $150 \mathrm{~nm}$. At $w$ of $100 \mathrm{~nm}$, the reduction in the $I_{\mathrm{D} \text {,max }}$ in multi-channel devices was as small as $12 \%$, much smaller than that in single-channel devices. This is because the multi-channel structure mitigates greatly the electronelectron and sidewall scatterings in tri-gate (MOS)HEMTs. In single-channel devices, electrons populate only one channel, resulting in a high electron density. In addition to a more pronounced electron-electron scattering, this reduces the effective distance between electrons and sidewalls, causing more sidewall scattering. Hence, $I_{\mathrm{D} \text {, max }}$ degrades rapidly with narrower fins. The multi-channel structure addresses this issue by better distributing electrons in multiple parallel channels, instead of only one, reducing the carrier density per channel and the effective distance between electrons and sidewalls, and thus mitigating the degradation and widthdependence of $I_{\mathrm{D} \text {,max }}$. This explanation is further supported by the dependence of $I_{\mathrm{D} \text {,max }}$ on $l$ [Fig. 4(b)]. Single-channel devices presented much larger and quicker degradation in

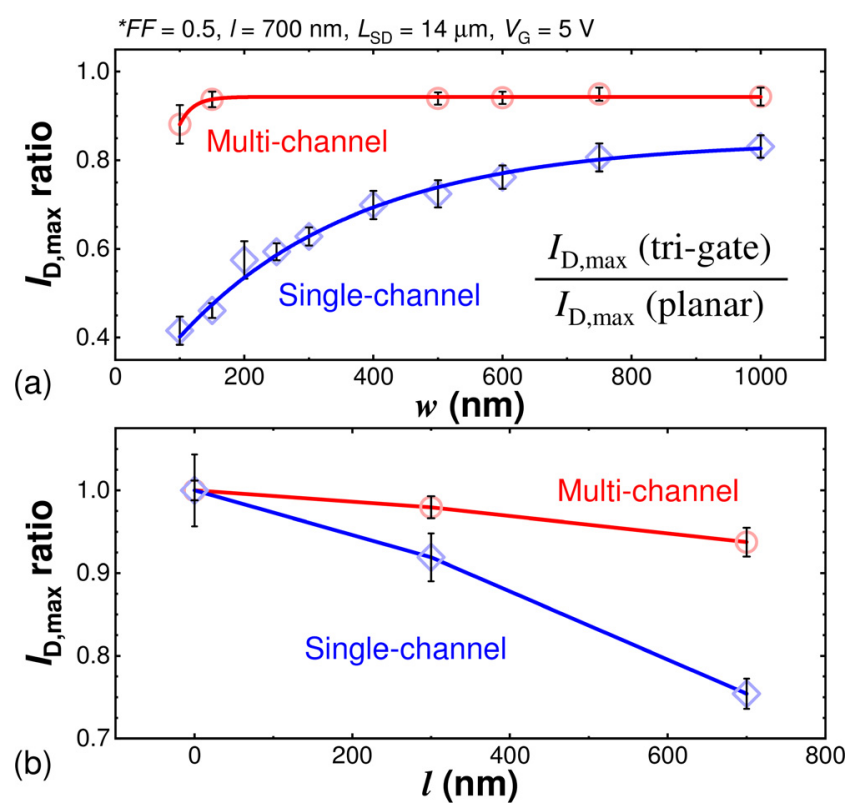

FIG. 4. Dependence of $I_{\mathrm{D}, \max }$ on (a) $w$ and (b) $l$ in single- and multi-channel tri-gate MOSHEMTs, all measured at $V_{\mathrm{G}}=5 \mathrm{~V}$ and normalized by the width of device footprint. These devices had different $w$ in their tri-gate regions, while sharing the same $F F$ of 0.5 and $l$ of $700 \mathrm{~nm}$. The single-channel devices in (b) had $F F$ of 0.64 . The distance between source and drain electrodes $\left(L_{\mathrm{SD}}\right)$ was $14 \mu \mathrm{m}$. 
$I_{\mathrm{D}, \max }$ as $l$ was increased, than in multi-channel devices, indicating the higher electron velocity in multi-channel devices due to the reduced scattering.

Based on these optimizations, we designed multichannel tri-gate GaN MOSHEMTs for high voltage applications. The $L_{\mathrm{GD}}$ was $10 \mu \mathrm{m}$ to sustain high voltages, and $l$ was $700 \mathrm{~nm}$ to decrease the area of etched regions on the 2DEG and reduce $R_{\mathrm{ON}}$. The fins were $100 \mathrm{~nm}$-wide, along with $s$ of $100 \mathrm{~nm}$ and $F F$ of $50 \%$. The gate metal was $2.5 \mu \mathrm{m}$ long, covering all the fins, and extended $0.5 \mu \mathrm{m}$ and $1.3 \mu \mathrm{m}$ towards the source and drain electrodes, respectively. Single-channel planar-gate and tri-gate GaN MOSHEMTs with similar dimensions were taken as the reference, based on a $20 \mathrm{~nm}$-thick $\mathrm{Al}_{0.25} \mathrm{GaN}$ barrier layer, which is a typical structure used for $\mathrm{GaN}$ power transistors.

The multi-channel tri-gate transistors presented significantly enhanced performance as compared with conventional single-channel tri-gate transistors. As shown in Fig. 5(a), the multi-channel tri-gate architecture reduced the $R_{\mathrm{ON}}$ from $11.2 \Omega \cdot \mathrm{mm}$ to $6.0 \Omega \cdot \mathrm{mm}$, and greatly increased the $I_{\mathrm{D}, \max }$ by more than 3.1 -fold, from $252 \mathrm{~mA} / \mathrm{mm}$ to $797 \mathrm{~mA} / \mathrm{mm}$ [all these measurements for both kinds of devices were normalized by the width of device footprint $(60 \mu \mathrm{m})]$. These results are remarkable since they indicate that the multi-channel trigate technology can lower the conduction losses of the transistor for a given device footprint, or equivalently, deliver a given current rating in a smaller device footprint, both of which are highly beneficial for efficient power transistors.

In addition, the multi-channel tri-gate architecture also addresses the degradation in $\mathrm{ON}$-state performance in singlechannel tri-gate transistors [Fig. 5(a)], such as the large $R_{\mathrm{ON}}$ and significantly diminished $I_{\mathrm{D}, \max }$ as compared to planargate transistors, which is mainly due to narrower effective

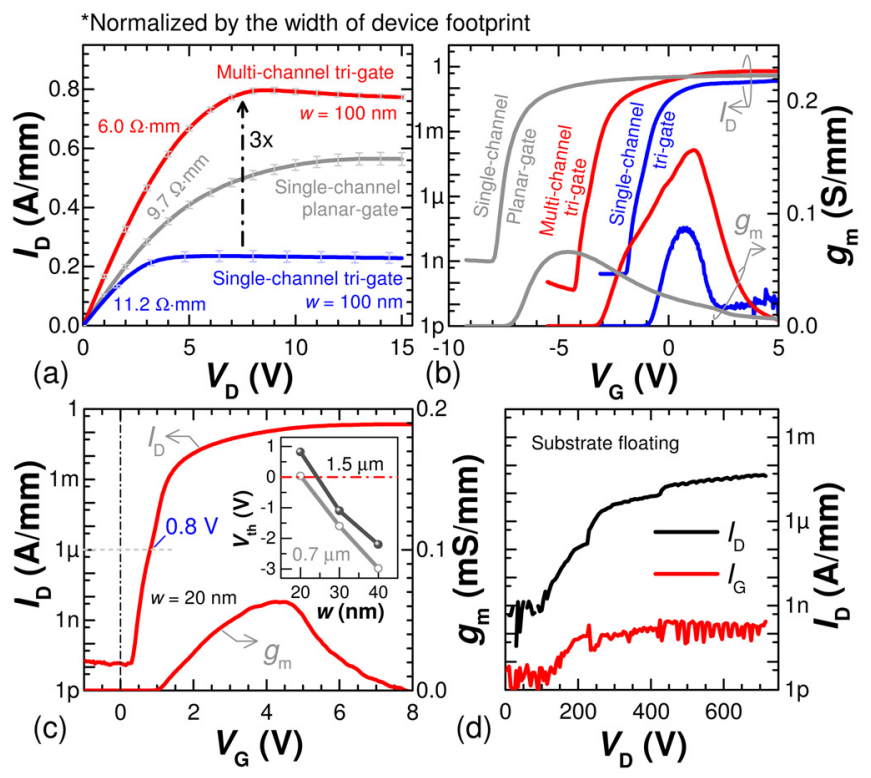

FIG. 5. (a) Output characteristics at $V_{\mathrm{G}}=5 \mathrm{~V}$ and (b) transfer characteristics at $V_{\mathrm{D}}=5 \mathrm{~V}$ of the transistors, normalized by the width of device footprint. The $w$ and $F F$ in single-channel tri-gate and multi-channel tri-gate transistors were $100 \mathrm{~nm}$ and $50 \%$, respectively. (c) Transfer characteristics of multichannel tri-gate transistors with $w$ of $20 \mathrm{~nm}$ and $F F$ of $10 \%$ at $V_{\mathrm{D}}=5 \mathrm{~V}$. (d) Typical OFF-state breakdown characteristics of the multi-channel tri-gate transistors measured with floating substrate. The inset in (c) shows the dependence of $V_{\mathrm{TH}}($ at $1 \mu \mathrm{A} / \mathrm{mm})$ in multi-channel tri-gate transistors on $w$ and $l$. channels, strain relaxation, and additional spreading resistance. $^{26-28}$ The multi-channel tri-gate architecture overcomes these issues thanks to the highly conductive parallel multi-channels with fewer carriers per channel. Compared with single-channel planar-gate transistors, $R_{\mathrm{ON}}$ was reduced by $38 \%$ and $I_{\mathrm{D}, \max }$ was increased by $41 \%$ in the multichannel tri-gate transistor, despite the $50 \%$ reduction in the effective channel width $(F F=50 \%)$.

The multi-channel tri-gate improves not only the output characteristics but also the transfer characteristics of the devices [Fig. 5(b)]. Compared to single-channel planar-gate devices, the $V_{\mathrm{TH}}$ in the multi-channel tri-gate transistor was reduced from $-7.6 \mathrm{~V}$ to $-3.6 \mathrm{~V}$, and $g_{\mathrm{m}, \max }$ was enhanced by 2.4-fold, from $66.1 \mathrm{mS} / \mathrm{mm}$ to $156.6 \mathrm{mS} / \mathrm{mm}$ in the multichannel tri-gate devices. The ON-state drain current $\left(I_{\mathrm{ON}}\right)$ was increased and the OFF-state $\left(I_{\mathrm{OFF}}\right)$ was diminished, resulting in a higher on/off ratio over $10^{10}$.

The multi-channel tri-gate architecture also provides a promising platform to achieve normally-off operation [Fig. 5(c)]. While typical methods developed for normally-off single-channel GaN transistors such as gate recess ${ }^{29}$ or $\mathrm{p}$ $\mathrm{GaN}^{30}$ may not deplete all embedded channels, the tri-gate offers a unique opportunity to use the sidewall-depletion effect $^{31}$ to deplete the multi-channels and achieve normallyoff operation. The sidewall-depletion effect originates from surfaces states at fin sidewalls, the large work function of the gate metals, and the elastic deformation of the fins that causes more strain relaxation in the $\mathrm{AlGaN} / \mathrm{GaN}$ heterostructure near the sidewalls, depleting the 2DEG in a certain width from the two sidewalls towards the center of the fin. When $w$ is equal to or smaller than the $\left(w_{\text {dep }}\right), 2 D E G$ in the multi-channel fins will be depleted and normally-off operation can thus be achieved. As shown in Fig. 5(c), by reducing $w$ to $20 \mathrm{~nm}(s=180 \mathrm{~nm})$, the $2 \mathrm{DEG}$ in the multi-channel fins was depleted and a positive $V_{\mathrm{TH}}$ of $0.82 \mathrm{~V}$ at $1 \mu \mathrm{A} / \mathrm{mm}$ was, along with $I_{\mathrm{OFF}}$ of only $12 \mathrm{pA} / \mathrm{mm}$ at $V_{\mathrm{G}}=0 \mathrm{~V}$, indicating excellent normally-off behavior. In addition, in this work we found that the $w_{\text {dep }}$ was $20 \mathrm{~nm}$ and $24 \mathrm{~nm}$ for $l$ of $700 \mathrm{~nm}$ and $1.5 \mu \mathrm{m}$, respectively [inset in Fig. 5(c)], which agrees well with other reports in the literature. ${ }^{32}$ The larger $w_{\text {dep }}$ for $l$ of $1.5 \mu \mathrm{m}$ is likely due to the greater strain relaxation within longer fins.

Despite the 3D nature of tri-gates, which leads to an increased surface area compared to conventional planar gates, the multi-channel tri-gate transistors showed very small gate leakage current of only about $0.2 \mathrm{nA} / \mathrm{mm}$ even at a high drain bias of $700 \mathrm{~V}$ when the transistors were in OFF state [Fig. 5(d)]. The devices presented a high hard breakdown $\left(V_{\mathrm{BR}}\right)$ of $715 \mathrm{~V}$, indicating the potential of the proposed technology for high-voltage applications.

In conclusion, we demonstrated high-voltage multichannel tri-gate GaN MOSHEMTs in this work, based on a unique combination of multi-channel $\mathrm{AlGaN} / \mathrm{GaN}$ heterostructures and tri-gate electrodes. A significant reduction in $R_{\mathrm{ON}}$ and enhancement of $g_{\mathrm{m}, \max }$ were demonstrated in normally-on devices. Normally-off operation was also achieved by sidewall-depletion effect, presenting a positive $V_{\mathrm{TH}}$ of $0.82 \mathrm{~V}$ at $1 \mu \mathrm{A} / \mathrm{mm}$ and a high $V_{\mathrm{BR}}$ of $715 \mathrm{~V}$. These results unveil a promising pathway for future efficient $\mathrm{GaN}$ power transistors with much reduced $R_{\mathrm{ON}}$. 
The authors would like to thank the staff in CMi and ICMP cleanrooms at EPFL for technical support. This work was supported in part by the European Research Council under the European Union's H2020 program/ERC Grant Agreement No. 679425 and in part by the Swiss National Science Foundation under the Assistant Professor Energy Grant Nos. PYAPP2_166901 and 200021_169362.

${ }^{1}$ K. J. Chen, O. Häberlen, A. Lidow, C. I. Tsai, T. Ueda, Y. Uemoto, and Y. Wu, IEEE Trans. Electron Devices 64, 779 (2017).

${ }^{2}$ E. A. Jones, F. F. Wang, and D. Costinett, IEEE J. Emerging Sel. Top. Power Electron. 4, 707 (2016).

${ }^{3}$ H. Lee, D. Piedra, M. Sun, X. Gao, S. Guo, and T. Palacios, IEEE Electron Device Lett. 33, 982 (2012).

${ }^{4}$ S. L. Selvaraj, A. Watanabe, A. Wakejima, and T. Egawa, IEEE Electron Device Lett. 33, 1375 (2012).

${ }^{5}$ J. Ma, M. Zhu, and E. Matioli, IEEE Electron Device Lett. 38, 1704 (2017).

${ }^{6}$ C. Ostermaier, G. Pozzovivo, J.-F. Carlin, B. Basnar, W. Schrenk, Y. Douvry, C. Gaquière, J.-C. DeJaeger, K. Čičo, K. Fröhlich, M. Gonschorek, N. Grandjean, G. Strasser, D. Pogany, and J. Kuzmik, IEEE Electron Device Lett. 30, 1030 (2009).

${ }^{7}$ T. Zimmermann, D. Deen, Y. Cao, J. Simon, P. Fay, D. Jena, and H. G. Xing, IEEE Electron Device Lett. 29, 661 (2008).

${ }^{8}$ T. Lim, R. Aidam, P. Waltereit, T. Henkel, R. Quay, R. Lozar, T. Maier, L. Kirste, and O. Ambacher, IEEE Electron Device Lett. 31, 671 (2010).

${ }^{9}$ J. Ma, X. Lu, X. Zhu, T. Huang, H. Jiang, P. Xu, and K. M. Lau, J. Cryst. Growth 414, 237 (2015).

${ }^{10}$ N. H. Sheng, C. P. Lee, R. T. Chen, D. L. Miller, and S. J. Lee, IEEE Electron Device Lett. 6, 307 (1985).

${ }^{11}$ G. W. Wang, Y. K. Chen, D. C. Radulescu, and L. F. Eastman, IEEE Electron Device Lett. 9, 4 (1988).

${ }^{12}$ H. Daembkes and G. Weimann, Appl. Phys. Lett. 52, 1404 (1988).

${ }^{13}$ Y. Cao, K. Wang, G. Li, T. Kosel, H. Xing, and D. Jena, J. Cryst. Growth 323, 529 (2011).
${ }^{14}$ S. Yamaguchi, M. Kosaki, Y. Wattanabe, Y. Yukawa, S. Nitta, H. Amano, and I. Akasaki, Appl. Phys. Lett. 79, 3062 (2001).

${ }^{15}$ S. Yamaguchi, Y. Iwamura, Y. Watanabe, M. Kosaki, Y. Yukawa, S. Nitta, S. Kamiyama, H. Amano, and I. Akasaki, Appl. Phys. Lett. 80, 802 (2002).

${ }^{16}$ S. Heikman, S. Keller, D. S. Green, S. P. DenBaars, and U. K. Mishra, J. Appl. Phys. 94, 5321 (2003).

${ }^{17}$ A. Terano, T. Tsuchiya, K. Mochizuki, S. Tanaka, and T. Nakamura, J. Jpn. Appl. Phys. 54, 066503 (2015).

${ }^{18}$ A. Kamath, T. Patil, R. Adari, I. Bhattacharya, S. Ganguly, R. W. Aldhaheri, M. A. Hussain, and D. Saha, IEEE Electron Device Lett. 33, 1690 (2012).

${ }^{19}$ J. Xue, J. Zhang, K. Zhang, Y. Zhao, L. Zhang, X. Ma, X. Li, F. Meng, and Y. Hao, J. Appl. Phys. 111, 114513 (2012).

${ }^{20}$ J. Wei, S. Liu, B. Li, X. Tang, Y. Lu, C. Liu, M. Hua, Z. Zhang, G. Tang, and K. J. Chen, IEEE Electron Device Lett. 36, 1287 (2015).

${ }^{21}$ R. Chu, Y. Zhou, J. Liu, D. Wang, K. J. Chen, and K. M. Lau, IEEE Trans. Electron Devices 52, 438 (2005).

${ }^{22}$ J. Ma, G. Santoruvo, P. Tandon, and E. Matioli, IEEE Trans. Electron Devices 63, 3614 (2016).

${ }^{23}$ J. Ma and E. Matioli, IEEE Electron Device Lett. 38, 1305 (2017).

${ }^{24}$ J. Ma and E. Matioli, Appl. Phys. Lett. 112, 052101 (2018).

${ }^{25}$ J. Ma, D. C. Zanuz, and E. Matioli, IEEE Electron Device Lett. 38, 1298 (2017).

${ }^{26}$ A. Dixit, A. Kottantharayil, N. Collaert, M. Goodwin, M. Jurczak, and K. D. Meyer, IEEE Trans. Electron Devices 52, 1132 (2005).

${ }^{27}$ M. Azize and T. Palacios, Appl. Phys. Lett. 98, 042103 (2011).

${ }^{28}$ J. Ma and E. Matioli, IEEE Electron Device Lett. 38, 367 (2017).

${ }^{29}$ W. Saito, Y. Takada, M. Kuraguchi, K. Tsuda, and I. Omura, IEEE Trans. Electron Devices 53, 356 (2006).

${ }^{30}$ S. Kaneko, M. Kuroda, M. Yanagihara, A. Ikoshi, H. Okita, T. Morita, K. Tanaka, M. Hikita, Y. Uemoto, S. Takahashi, and T. Ueda, in 2015 IEEE 27 th International Symposium on Power Semiconductor Devices and IC's (ISPSD), Hong Kong (2015), p. 41.

${ }^{31}$ G. Santoruvo, A. Allain, D. Ovchinnikov, and E. Matioli, Appl. Phys. Lett. 109, 103102 (2016).

${ }^{32}$ E. Matioli and T. Palacios, Nano Lett. 15, 1070 (2015). 